\title{
Combined pericarditis and pneumonia caused by Legionella infection
}

\author{
JESPER HASTRUP SVENDSEN, VIGGO JØNSSON, UFFE NIEBUHR \\ From the Department of Cardiology, Department of Internal Medicine and Department of Thoracic Surgery, \\ Gentofte Hospital, University of Copenhagen, Denmark
}

SUMMARY During a one year period acute pericarditis was diagnosed in 16 consecutive patients without acute infarction or malignancy. In two of these patients with both pericarditis and pneumonia Legionella infection was present. One case was caused by Legionella longbeachae and the other by both Legionella longbeachae and Legionella jordanis. When pericarditis is associated with pneumonia Legionella infection should be sought so that effective treatment with erythromycin may be started early.

Pneumonia caused by Legionella pneumophila has been recognised since $1976 .^{1}$ Legionella infection may range from a mild fever with seroconversion to the manifest Legionnaires' disease with severe pneumonia, sepsis, and several extrapulmonary manifestations, ${ }^{23}$ including both myocarditis ${ }^{4}$ and pericarditis. $^{5-7}$

We describe two cases of severe exudative pericarditis in patients with pneumonia caused by Legionella infection and we emphasise the importance of separating the Legionella pericarditis from other causes of acute pericarditis so that treatment with erythromycin can be started early. The two cases were found among 16 pericarditis patients admitted to our hospital in the past year without acute myocardial infarction or malignant disease.

\section{Case reports}

\section{PATIENT A}

A 61 year old man, who had been in good health, was admitted with fever, non-productive cough, and headache. On admission his temperature was $40.0^{\circ} \mathrm{C}$. The pulse was regular $(84$ beats $/ \mathrm{min})$ and the blood pressure was $150 / 80 \mathrm{~mm} \mathrm{Hg}$. A pericardial friction rub was heard. There were no signs of right ventricular failure. Repeated chest $x$ ray showed diffuse fine shadowing of the left lower pulmonary lobe and small bilateral pleural effusions. The con-

Requests for reprints to Dr Jesper Hastrup Svendsen, Department of Internal Medicine B, Rigshospitalet, Blegdamsvej, DK-2100 Copenhagen, Denmark.

Accepted for publication 9 July 1987 tour of the heart was normal. Cross sectional echocardiography showed a pericardial effusion with an echo free zone measuring $12 \mathrm{~mm}$ in diameter.

The leucocyte count was $12.9 \times 10^{9} / 1$ with $71 \%$ neutrophils. After blood had been taken for culture he was put on intravenous penicillin ( 2 million units four times a day) and netilmicin ( $150 \mathrm{mg}$ three times a day) without effect. Because a weak positive Legionella titre was detected the antibiotic treatment was changed to intravenous erythromycin $1 \mathrm{~g}$ four times a day). Four days later oral rifampicin $(300 \mathrm{mg}$ three times a day) was added because a low grade fever persisted. The patient improved and his temperature became normal. Nineteen days after admission the chest $x$ ray was normal and echocardiography showed only a minor pericardial effusion.

Blood cultures showed no bacterial growth. Legionella longbeachae titres were 32 on day 2, 2,000 on day 14, 512 on day 45, and 256 on day 73 .

\section{PATIENT B}

A 44 year old previously healthy man was admitted with acute chest pain. On admission the temperature was normal, blood pressure $110 / 80 \mathrm{~mm} \mathrm{Hg}$, and the pulse rate was 60 beats $/ \mathrm{min}$. A pericardial friction rub was heard. No signs of right ventricular failure was seen. Electrocardiography showed minor ST segment elevations suggesting acute pericarditis. Chest $x$ ray revealed cardiac enlargement but no lung shadows. The patient was discharged after a few days without symptoms and without audible friction rub. The cause of the pericarditis was not 
sought. Six months later he was readmitted with identical chest discomfort without fever. Again a pericardial friction rub was heard. White blood cell counts were $13.4 \times 10^{9} / 1$ and the erythrocyte sedimentation rate was $36 \mathrm{~mm} / \mathrm{h}$. The chest $x$ ray was unchanged without signs of pneumonia. Cross sectional echocardiography revealed a pericardial effusion with an echo free zone measuring $5 \mathrm{~mm}$ anteriorly and $10 \mathrm{~mm}$ posteriorly.

Two weeks later, echocardiography showed that the size of the pericardial effusion had increased considerably and in addition paradoxicat movement of the interventricular septum was seen. A pericardial fenestration improved the condition considerably. Sixteen days after the fenestration the patient again had left-sided chest pain and his temperature rose to $38.5^{\circ} \mathrm{C}$. The chest $x$ ray now showed an infiltrate in the left lower pulmonary lobe and a left pleural effusion. Pulmonary embolism was ruled out. The white blood cell count was $10.8 \times 10^{9} / 1$ with $76 \%$ neutrophils. Treatment with intravenous ampicillin ( $1 \mathrm{~g}$ four times a day) had no effect on fever while oral pivampicillin $(700 \mathrm{mg}$ four times a day) reduced his temperature to normal. Culture of blood and pleural fluid showed no bacterial growth. Yersinia, Mycoplasma pneumoniae, cytomegalovirus, influenza virus, adeno virus, and HIV infection were not found. On day 32 Legionella titres were: $L$ longbeachae 256 and $L$ jordanis 256 and on day 40: $L$ longbeachae 256 and $L$ jordanis 512 .

He was discharged on day 47 free of symptoms, with a normal temperature, a normal white blood cell count, and a normal erythrocyte sedimentation rate. He was not treated with erythromycin during the course of the illness.

\section{Discussion}

Legionella infection is a rare cause of acute pericarditis. In 16 patients with acute pericarditis admitted within the past year we found two cases in patients with associated pneumonia and serological signs of Legionella infection with $L$ longbeachae and $L$ jordanis. Unlike $L$ pneumophila, these species have not previously been associated with cardiac disease in Legionella infection.

Unfortunately the pericardial fluid from case B was not tested for Legionella; however, Maycock et al cultured Legionella from the pericardial fluid ${ }^{7}$ and confirmed that pericarditis in their case was of a primary bacteriological origin.

Three of the four previously described cases of Legionella pericarditis had a fever with a temperature of less than $39.0^{\circ} \mathrm{C} .5^{58}$ Two had pericardial tamponade. ${ }^{57}$ One of our cases (A) had high grade fever whereas the temperature was normal in the other case. Both our cases had pulmonary shadowing and pleural effusions. Case $B$ had antibody titres consistent with Legionella infection at the time of the acute episodes of pericarditis. The pattern of the Legionella titres does not suggest that the patient caught the infection at the time of fenestration when pulmonary infiltrates were present at an early stage of the disease.

Legionella pericarditis should be suspected in cases of combined pericarditis and pneumonia. Prompt identification of Legionella will permit early appropriate treatment with erythromycin and this may prevent the development of long standing disease that was seen in our patients.

\section{References}

1 Fraser DW, Tsai TR, Orenstein W, et al. Legionnaires' disease: description of an epidemic of pneumonia. N Engl J Med 1977;297:1189-97.

2 Tsai TF, Finn OR, Plikaytis BD, McCauley W, Martin SM, Fraser DW. Legionnaires' disease: clinical features of the epidemic in Philadelphia. Ann Intern Med 1979;90:509-17.

3 Swartz MN. Clinical aspects of Legionnaires' disease. Ann Intern Med 1979;90:492-5.

4 Gross D, Willens H, Zeldis SM. Myocarditis in Legionnaires' disease. Chest 1981;79:232-4.

5 Harris LF. Legionnaires' disease associated with massive pericardial effusion [Letter]. Arch Intern Med 1981;141:1385.

6 Landes BW, Pogson W, Beauchamp GD, Skillman RK, Brewer JH. Pericarditis in a patient with Legionnaires' disease. Arch Intern Med 1982;142: 1234-5.

7 Mayock R, Skale B, Kohler RB. Legionella pneumophila pericarditis proved by culture of pericardial fluid. Am J Med 1983;75:534-6.

8 Friedland L, Snydman DR, Weingarden AS, Hedges TR, Brown R, Busky M. Ocular and pericardial involvement in Legionnaires' disease. Am J Med 1984;77:1105-7. 